rates $\mathrm{n}=25(73,5 \%)$ than those with complete pathologic response $n=9(26,5 \%)(p=0,006)$. Surgery performed until 6 months after CRT reduced recurrences in the first 5 years of follow up $(p=0,01)$. Among patients submitted to surgery with $\mathrm{RD}, 89,5 \%(\mathrm{n}=17 / 19)$ presented distant metastasis during follow up $(\mathrm{p}=0,03)$. Multivariated analysis showed $\mathrm{RD}$ as a predictive factor for recurrence $(\mathrm{p}=0,02, \mathrm{HR}=1,85 \mathrm{CI}$ $(1,07-3,19))$. DFS and OS was not significantly different between surgery and control group (log rank test, $\mathrm{p}=0,25$ and $\mathrm{p}=0,13$, respectively). In multivariate analysis, overall survival was found to be associated with $\mathrm{RD}(\mathrm{p}=0,001)$ and recurrence $(\mathrm{p}<0,001)$.

Conclusions Completion surgery after CRT highlights the pathologic response as a prognostic factor. It cannot be accessed with accuracy by physical exam, imaging or biopsy and is associated with recurrence and death bringing information that can be used to tailor further treatment.

\section{EPV042/\#199 RACIAL AND REGIONAL DISPARITIES IN THE DIAGNOSIS OF ADVANCED STAGE CERVICAL CANCERS IN THE US: WHO IS MOST AT RISK?}

${ }^{1} \mathrm{C}-\mathrm{IL}$ Liao, ${ }^{2} \mathrm{~A}$ Francoeur*, ${ }^{2} \mathrm{D}$ Wong, ${ }^{3} \mathrm{~A}$ Mann, ${ }^{4} \mathrm{MA}$ Caesar, ${ }^{5} \mathrm{~A}$ Chan, ${ }^{6} \mathrm{~B}$ Monk, ${ }^{7} \mathrm{D}$ Kapp, ${ }^{8} \mathrm{~J}$ Chan. ${ }^{1}$ Kaohsiung Veterans General Hospital, Obstetrics and Gynecology, Kaohsiung City, Taiwan; ' University of California Los Angeles, Obstetrics and Gynecology, Los Angeles, USA; ${ }^{3}$ Palo Alto Medical Foundation, Research Institute, Palo Alto, USA; ${ }^{4}$ California Pacific Medical Center, Research Institute, San Francisco, USA; ${ }^{5}$ Palo Alto Medical Foundation Research Intitute, Obstetrics and Gynecology, Palo Alto, USA; ${ }^{6}$ Arizona Oncology, Gynecologic Oncology, Obstetrics and Gynecology, Phoenix, USA; ${ }^{7}$ Stanford University School of Medicine, Department of Radiation Oncology, Stanford, USA; ${ }^{8}$ California Pacific Medical Center, Obstetrics and Gynecology, San Francisco, USA

\subsection{6/ijgc-2021-IGCS.110}

Objectives Prior studies have found an increase in advanced stage cervical cancers in the US. We propose to determine the high risk group based on demographic and clinical characteristics.

Methods Microscopic confirmed cervical cancer was obtained from United States Cancer Statistics (USCS) from 2001 to 2017. Age-adjusted incidence (AAI, per 100,000 women, corrected by US 2000 standard population), age-specific incidence (ASI, per 100,000 women), and trends were calculated by SEER*Stat 8.3.8 and Joinpoint Regression Program 4.8.0.1.

Results Of 27,102 patients with advanced stage cervical cancer from 2001-2017, 17,097 (63\%) were White, 4,939 (5\%) were Black, 3,636 were Hispanic (2\%), and 1,117 were Asian (0.5\%). Squamous and adenocarcinoma consists of 17,867 and 4,992 patients, respectively. The age group with the highest incidence of advanced cancer was 50-54 years, 2.29/100,000. Based on race, Black and Hispanic patients have higher incidence at $1.35 / 100,000$ and $1.18 / 100,000$ compared to White patients, 0.86/100,000. With respect to region, the South has the greatest incidence at 1.04/100,000. The intersectionality of age, race and region finds that Black women, aged 65-69, residing in the South have the highest incidence at $4.19 / 100,000$, an incidence nearly three times higher than White women of the same age in the South at only 1.63/100,000.

Conclusions Advanced stage cervical cancer continues to disproportionately affect minorities in Southern regions in the US. Resources toward screening and vaccination are needed in these at risk groups.

\section{EPV043/\#200 THE INCREASING INCIDENCE OF METASTATIC CERVICAL CANCER IN THE UNITED STATES - WHAT FACTORS ARE RESPONSIBLE?}

${ }^{1} \mathrm{C}-\mathrm{I}$ Liao, ${ }^{2} \mathrm{~A}$ Francoeur, ${ }^{3} \mathrm{~A}$ Milki, ${ }^{4} \mathrm{E}$ Thayer, ${ }^{5} \mathrm{~A}$ Mann, ${ }^{6} \mathrm{MA}$ Caesar, ${ }^{7} \mathrm{~A}$ Chan, ${ }^{8} \mathrm{~B}$ Monk, ${ }^{9} \mathrm{D}$ Kapp, ${ }^{10} \mathrm{~J}$ Chan. ${ }^{1}$ Kaohsiung Veterans General Hospital, Obstetrics and Gynecology, Kaohsiung City, Taiwan; ' ${ }^{2}$ nniversity of California Los Angeles, Obstetrics and Gynecology, Los Angeles, USA; ${ }^{3}$ George Washington University School of Medicine and Health Sciences, Obstetrics and Gynecology, Washington DC, USA; ${ }^{4}$ University of Massachusetts Medical School, Obstetrics and Gynecology, Worcester, USA; ${ }^{5}$ Palo Alto Medical Foundation, Research Institute, Palo Alto, USA; ${ }^{6}$ California Pacific Medical Center, Research Institute, San Francisco, USA; ${ }^{7} \mathrm{Pal}$ o Alto Medical Foundation Research Intitute, Obstetrics and Gynecology, Palo Alto, USA; ${ }^{8}$ Arizona Oncology, Gynecologic Oncology, Obstetrics and Gynecology, Phoenix, USA; ${ }^{9}$ Stanford University School of Medicine, Department of Radiation Oncology, Stanford, USA; ${ }^{10}$ California Pacific Medical Center, Obstetrics and Gynecology, San Francisco, USA

\subsection{6/ijgc-2021-IGCS.111}

Objectives To determine the incidence and trends of advanced stage cervical cancer in the United States.

Methods Data were obtained from the U.S. Cancer Statistics program from 2001-2017. SEER*Stat 8.3.8 and Joinpoint regression program 4.8.0.1 were used to calculate incidence trends.

Results of 27,102 patients with advanced stage cervical cancer from 2001-2017, 17,097 (63\%) were White, 4,939 (5\%) were Black, 3,636 were Hispanic (2\%), and 1,117 were Asian $(0.5 \%)$. Over time, there has been an annual increase in advanced stage cervical cancer at a rate of nearly $2 \%$ per year $(\mathrm{p}<0.001)$; however, those with early stage cancers have a decrease of $1.54 \%$ annually $(p<0.001)$. Women aged 30 to 65 years showed an overall increase in incidence, however those 30-34 years olds have a particularly high increase at 3.39\% annually $(\mathrm{p}<0.001)$. Although the overall incidence of advanced cancers is higher in Hispanic and Black populations, there is an increasing number of new cases in White women at $2.39 \%$ annually $(\mathrm{p}<0.001)$. Compared to other groups, the intersection of White women aged $40-44$ in the South have the highest average annual increase at 5.07\% $(\mathrm{p}<0.001)$.

Conclusions Although the overall incidence of advanced cervical cancers is highest in Hispanic and Black women, there is an increase in incidence in White women particularly in the Southern region of the U.S. More research is needed to understand this trend particularly in relation to screening and treatment of precancerous disease.

\section{EPV044/\#201 UNDERSTANDING THE NEVER-SCREENED POPULATION FOR CERVICAL CANCER IN THE UNITED STATES - A DESCRIPTIVE AND TREND ANALYSIS}

${ }^{1} \mathrm{~A}$ Francoeur*, ${ }^{2} \mathrm{C}-\mathrm{I}$ Liao, ${ }^{1} \mathrm{D}$ Wong, ${ }^{3} \mathrm{E}$ Thayer, ${ }^{4} \mathrm{~A}$ Milki, ${ }^{5} \mathrm{~A}$ Moon, ${ }^{6} \mathrm{~A}$ Mann, ${ }^{7} \mathrm{MA}$ Caesar, ${ }^{8} \mathrm{~A}$ Chan, ${ }^{9} \mathrm{D}$ Kapp, ${ }^{10} \mathrm{~B}$ Monk, ${ }^{11} \mathrm{~J}$ Chan. ${ }^{1}$ University of California Los Angeles, Obstetrics and Gynecology, Los Angeles, USA; ${ }^{2}$ Kaohsiung Veterans General Hospital, Obstetrics and Gynecology, Kaohsiung City, Taiwan; ${ }^{3}$ University of Massachusetts Medical School, Obstetrics and Gynecology, Worcester, USA; ${ }^{4}$ George Washington University School of Medicine and Health Sciences, Obstetrics and Gynecology, Washington DC, USA; ${ }^{5}$ Stanford University School of Medicine, Obstetrics and Gynecology, Division of Gynecologic Oncology, Stanford, USA; ${ }^{6}$ Palo Alto Medical Foundation, Research Institute, Palo Alto, USA; ${ }^{7}$ California Pacific Medical Center, Research Institute, San Francisco, USA; ${ }^{8}$ Palo Alto Medical Foundation Research Intitute, Obstetrics and Gynecology, Palo Alto, USA; ${ }^{9}$ Stanford University School of Medicine, Department of Radiation Oncology, Stanford, USA; ${ }^{10}$ Arizona Oncology, Gynecologic Oncology, Obstetrics and Gynecology, Phoenix, USA; ${ }^{11}$ California Pacific Medical Center, Obstetrics and Gynecology, San Francisco, USA

10.1136/ijgc-2021-IGCS.112 